\title{
Lipoprotein(a) and mortality—a high risk relationship
}

\author{
Reinhard Klingel ${ }^{1,2} \cdot$ Andreas Heibges $^{1} \cdot$ Cordula Fassbender $^{1}$
}

Published online: 5 March 2019

(c) The Author(s) 2019

\begin{abstract}
Lipoprotein(a) (Lp(a)) is an independent cardiovascular risk factor playing a causal role for atherosclerotic cardiovascular disease (ASCVD). Early or progressive ASCVD or a familial predisposition are key findings which can be associated with Lp(a)-hyperlipoproteinemia (Lp(a)-HLP). The German guideline for the indication of lipoprotein apheresis in patients with $\mathrm{Lp}(\mathrm{a})$-HLP has proved to be of value to identify patients at highest risk, using the composite of a Lp(a) threshold $>60 \mathrm{mg} / \mathrm{dl}$ $(>120 \mathrm{nmol} / \mathrm{l})$ and clinical ASCVD progression despite effective LDL-C lowering therapy. In particular for such patients it appears to be plausible that $\mathrm{Lp}(\mathrm{a})$-associated risk would increase cardiovascular mortality as the most important part of total mortality in Western populations. By the majority of existing investigations an association of Lp(a) concentration on total or cardiovascular mortality was demonstrated. However, inconsistency in the findings between studies exists without a clear trend for any study feature to explain this. Genetic homogeneity of the population, long-term follow-up, and clinically guided selection of patients might be important to further clarify the impact of $\mathrm{Lp}$ (a) concentration on progression of ASCVD, and finally total or cardiovascular mortality. LDL and $\mathrm{Lp}(\mathrm{a})$ particles exhibit a mutual effect modification on related ASCVD risk. Therefore, LDL-C levels and concomitant LDL-C lowering treatment must be considered in this context. Prospective evaluation is needed to document that specific Lp(a)-lowering additional to targeted LDL-C lowering will in fact reduce cardiovascular or total mortality.
\end{abstract}

Keywords Lipoprotein (a) · Atherosclerosis · Cardiovascular disease $\cdot$ Mortality

\section{Background}

Atherosclerotic cardiovascular diseases (ASCVD) are recognized as the leading causes of death in Western countries and increasingly worldwide. The majority of these ASCVD deaths are attributable to either coronary heart disease (CHD) or cerebrovascular disease. It was demonstrated already in 1981 that Lipoprotein(a) (Lp(a)) concentrations above $30 \mathrm{mg} / \mathrm{dl}$ are associated with an increasing risk for myocardial infarction (MI) in genetically homogeneous Caucasian populations [1,2]. The Copenhagen City Heart Study was ground-breaking in retrieving attention to the clinical relevance of $L p(a)$ for ASCVD especially MI in the general population [3-5]. Above the 90th percentile (in this study $>85 \mathrm{mg} / \mathrm{dl}$ ), relative risk was about 3-fold higher.

This article is part of the special issue "Lp(a) - Update 2018"

Reinhard Klingel

klingel@apheresis-research.org

1 Apheresis Research Institute, Stadtwaldguertel 77, 50935 Cologne, Germany

2 1st Department of Internal Medicine, University of Mainz, Mainz, Germany
However, $\mathrm{Lp}$ (a) associated absolute 10-year risk of MI and CHD was not a constant factor of ASCVD but increased from women to men, and with smoking, hypertension, or increasing age [3]. Above $50 \mathrm{mg} / \mathrm{dl}$ the curve of $\mathrm{Lp}(\mathrm{a})$ and ASCVD risk association follows an almost linear fashion. At low $\mathrm{Lp}$ (a) concentrations, up to $30 \mathrm{mg} / \mathrm{dl}$ and the grey zone between concentrations of 30 to $50 \mathrm{mg} / \mathrm{dl}, \mathrm{Lp}(\mathrm{a})-\mathrm{me}$ diated risk seems to be clinically less important, putatively because low-density lipoprotein cholesterol (LDL-C) mediated risk, caused by the far more numerous regular LDL particles prevails [6]. As a result of this relationship and despite the lack of controlled intervention studies, the European Atherosclerosis Consensus Panel formulated a desirable range for $\mathrm{Lp}$ (a) below $<50 \mathrm{mg} / \mathrm{dl}$ [7]. The causal relationship has subsequently been reinforced by epidemiological studies, meta-analyses and Mendelian randomization studies $[4,5]$. In addition to CHD, there is also an association with peripheral arterial occlusive disease, stroke, and calcific aortic stenosis $[5,8]$.

$\mathrm{Lp}(\mathrm{a})$ is a predictor of incident or recurrent ASCVD complications. Elevated Lp(a) was shown to be a risk factor for clinical recurrence four to six months after coronary angioplasty. A median Lp(a) of $61 \mathrm{mg} / \mathrm{dl}$ in the highest quintile had a twofold higher recurrence rate compared to the median $\mathrm{Lp}(\mathrm{a})$ of $2-9 \mathrm{mg} / \mathrm{dl}$ in the two lowest quintiles [9]. 
Using $25 \mathrm{mg} / \mathrm{dl}$ as cut-off point a highly significant impact of $\mathrm{Lp}$ (a) on the long-term prognosis after successful coronary stenting was shown by the three year follow-up of 483 consecutive patients with acute coronary syndromes [10]. The Pro(a)LiFe trial studied 170 patients with Lp(a)-hyperlipoproteinemia (Lp(a)-HLP) and progressive ASCVD over a median period of 7.6 years since diagnosis of ASCVD before commencing lipoprotein apheresis (LA), followed by a prospective period of 5 years during regular LA, which resulted in stabilization of ASCVD [11]. Mean $\mathrm{Lp}$ (a) concentration before the onset of LA was $108 \mathrm{mg} / \mathrm{dl}$. Mean corrected LDL-C was $66.3 \mathrm{mg} / \mathrm{dl}$ in the two years before initiation of regular LA with lipid lowering medication, documenting that patients were in their risk-adjusted LDL$\mathrm{C}$ target range. The potential effect of attenuating ASCVD risk associated with elevated $\mathrm{Lp}$ (a) by decreasing LDL-C levels had been fully utilized in these patients before initiation of LA. The question whether cardiovascular mortality is increased due to Lp(a)-HLP in the period of progressive ASCVD remained unanswered because with this design only patients were studied, who were alive when the $\mathrm{Lp}$ (a) associated ASCVD was diagnosed. With all this information it appears to be plausible that Lp(a)-HLP has an impact on cardiovascular or total mortality.

\section{ASCVD and mortality}

Temporal changes in clinical care must be considered as potential confounders in this context, including interventional cardiology and secondary prevention measures beyond lipid lowering, such as antiplatelet therapies and antihypertensive therapies [12]. Consistent evidence has shown that statins reduce total cardiovascular events, and that further lowering of LDL-C levels by intensifying statin therapy, adding ezetimibe, or adding a proprotein convertase subtilisin/kexin type 9 (PCSK9)-inhibiting monoclonal antibody provides incremental additional reduction in cardiovascular risk [13-15]. However, there is less consistency in the magnitude of the reductions in individual mortality and cardiovascular end points among the various trials. In a meta-analysis of the impact of LDL-C lowering on total and cardiovascular mortality the magnitude of benefit appeared to decrease as the mean LDL-C levels of patients decreased [14]. The analysis also found an apparent lower limit for mortality benefit. Trials that enrolled patients with low baseline LDL-C levels, e. g. $<100 \mathrm{mg} / \mathrm{dl}$, did not show a mortality benefit. The $4 \mathrm{~S}$ trial enrolled patients with a mean baseline LDL-C of $188 \mathrm{mg} / \mathrm{dl}$ more than 2 decades ago, when mortality for acute MI was significantly higher than it is today. In the $4 \mathrm{~S}$ trial acute MI was fatal in $16.3 \%$ in the control group and $12.5 \%$ in the simvastatin group. In contrast, in the contemporary IMPROVE-IT and FOURIER trials mean baseline LDL-C levels were 93.8 and $92 \mathrm{mg} / \mathrm{dl}$, respectively $[13,15]$. Acute MI mortality was only 4.7 and $4.4 \%$ in control groups, and 4.2 and $5.3 \%$ in the ezetimibe and evolocumab groups.

\section{LDL and $L p(a)$ particles exhibit mutual ASCVD risk effect modification}

Lp(a) consists of an LDL particle whose apolipoprotein B is covalently bound to apolipoprotein(a) $[4,5]$. The physiological function of $\mathrm{Lp}(\mathrm{a})$ is still poorly understood. Although the hazard ratios in the Copenhagen City Heart Study showed an invariable relative risk increase when comparing $\mathrm{Lp}$ (a) $\geq 80^{\text {th }}$ cohort percentile with $\mathrm{Lp}$ (a) $<80$ th cohort percentile, the absolute risk characterizing a patient with elevated Lp(a) depended on the LDL-C level of that subject [6]. Given levels of $L p(a)$ combined with different decreasing corrected LDL-C resulted in corresponding decreased $L p(a)$ associated relative risk. In view of the overwhelming evidence on LDL-C lowering therapies and decrease in ASCVD risk independent from treatment modality, these observational data imply that aggressive lowering of LDL-C in individuals with high Lp(a) could divert a substantial part of the adverse effects of $L p(a)$, thereby markedly reducing the absolute ASCVD risk at least in a primary prevention setting [6]. Intervention studies for primary as well as secondary prevention showed that $L p(a)$ retains its character as a risk factor, even when the target range of LDL-C $<70 \mathrm{mg} / \mathrm{dl}$ has been achieved in manifested ASCVD. In subgroup analyses of the IMPROVEIT study with ezetimibe, the AIM-HIGH study with nicotinic acid, the JUPITER study with rosuvastatin and the LIPID study with pravastatin, a residual $L p(a)$-associated risk was detected despite effectively treated LDL-C [5]. Increased hazard ratio of cardiovascular events reflecting residual risk was documented in particular at $\mathrm{Lp}$ (a) concentrations exceeding $50 \mathrm{mg} / \mathrm{dl}$ when LDL-C was effectively lowered with statins [16]. This finding is in accord with previous suggestions to control cardiovascular risk factors and prescribe physical activity and a healthy diet as pivotal for the management of patients with high $\operatorname{Lp}(\mathrm{a})$, although $\mathrm{Lp}$ (a) concentration itself cannot be influenced by diet or lifestyle [17].

\section{Investigations on Lp(a) and mortality}

Several studies investigated a potential impact of $L p(a)$ on cardiovascular or total mortality. Key features of these studies are summarized in Table 1. Only studies were selected which were almost entirely composed of Caucasians, which is important as the distribution of $L p(a)$ isoforms and 


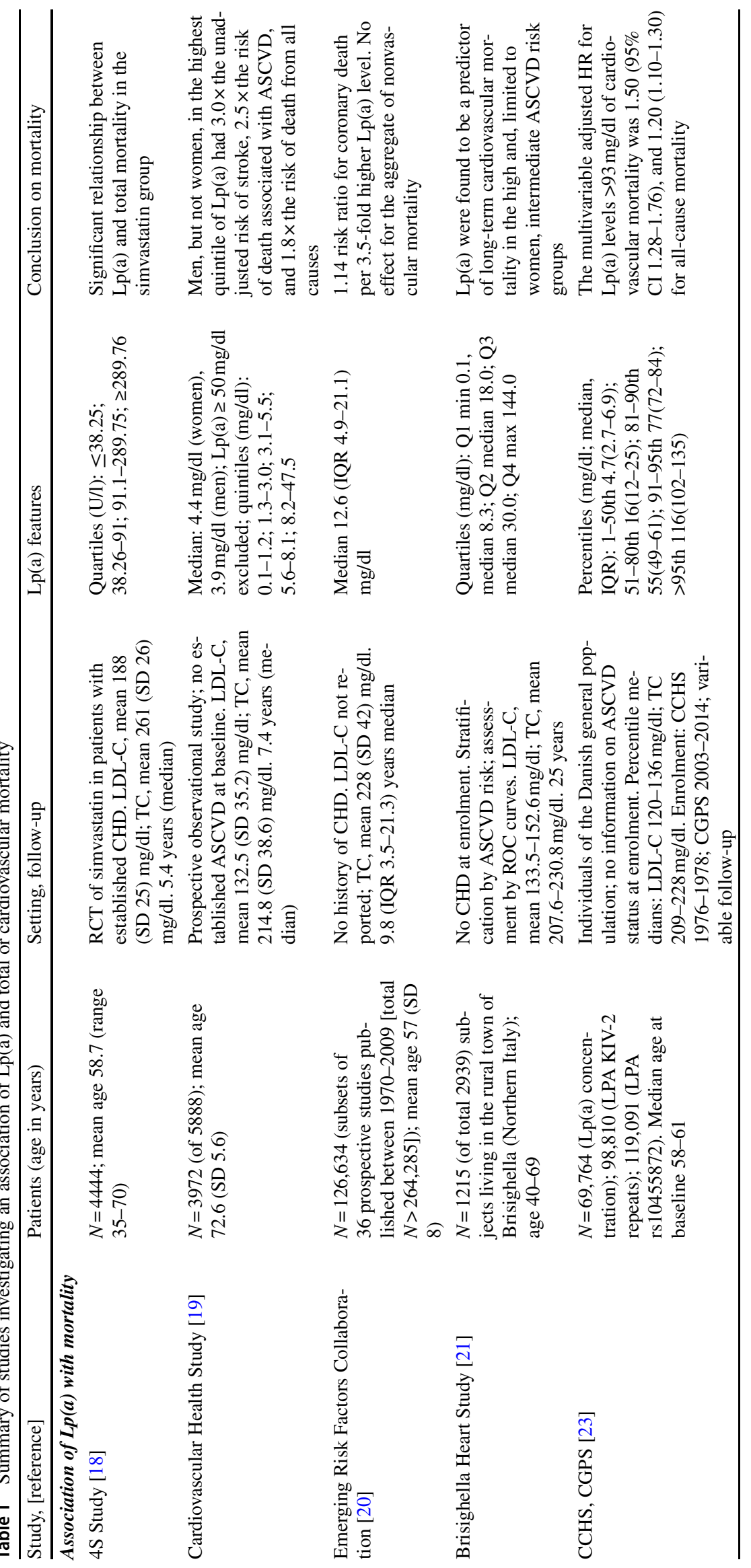




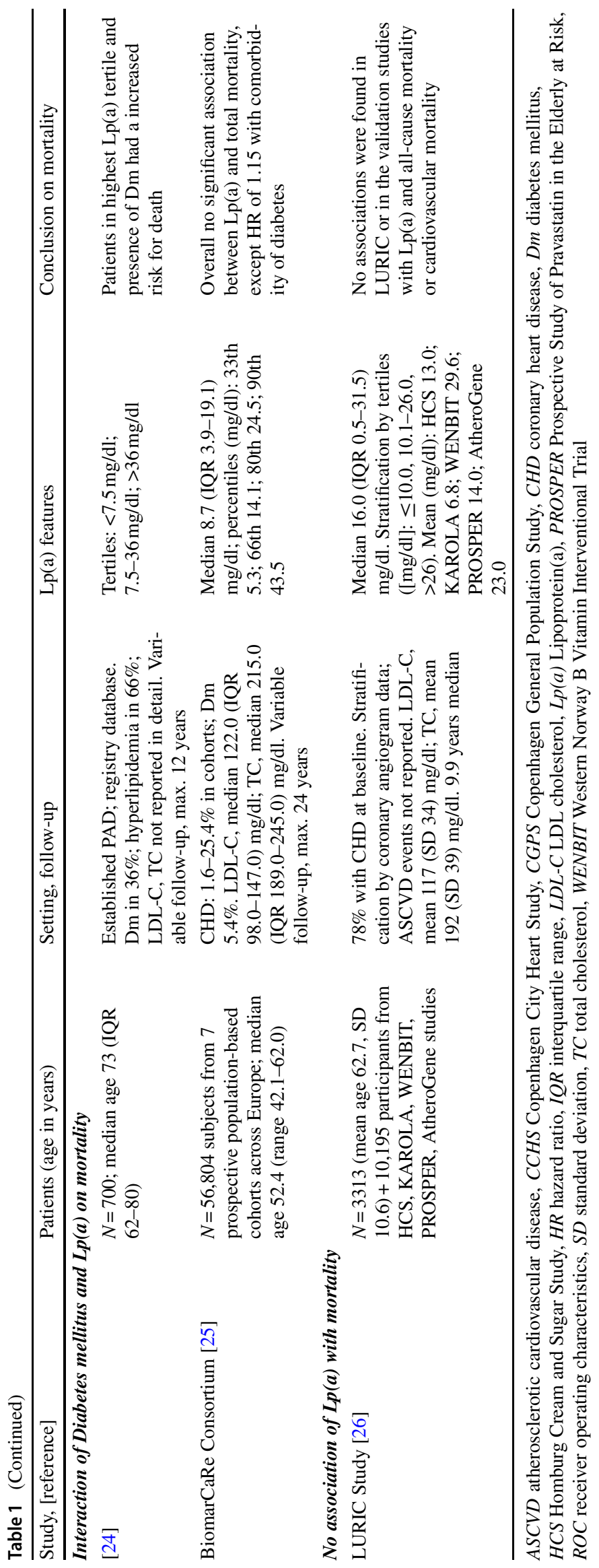


plasma $\mathrm{Lp}$ (a) concentrations is determined by ethnicity. The Scandinavian Simvastatin Survival Study (4S) was the first prospective study to show that cholesterol reduction in patients with CHD reduces total mortality [18]. Mean Lp(a) concentrations were significantly higher in patients with than in patients without major coronary events in the Simvastatin group, the placebo group, and both groups combined. Logistic regression analyses clearly confirmed that there was a significant relationship between $L p(a)$ concentration and mortality as well as major coronary events, the risk in both cases increasing with increasing Lp(a) concentration [18].

In the prospective Cardiovascular Health Study of adults 65 years of age or older in the United States, who were free of ASCVD, elevated Lp(a) concentration was an independent predictor of stroke, death from vascular disease, and death from any cause in men but not in women [19]. The risk associated with each quintile level of $L p(a)$ was determined using Cox proportional-hazards models with the lowest quintile serving as the reference group. As compared with those in the lowest quintile, men in the highest quintile had almost three times the risk of death associated with vascular events (relative risk, 2.54; 95\% confidence interval (CI), 1.59 to 4.08 ), and nearly twice the risk of death from all causes (relative risk, 1.76; 95\% CI, 1.31 to 2.36). In general elevated $\mathrm{Lp}$ (a) confers the same cardiovascular risk in women as in men as proved by epidemiological and Mendelian randomization studies.

The Emerging Risk Factors Collaboration assessed the relationship of $L p(a)$ concentration with risk of major vascular and nonvascular outcomes. Long-term prospective studies that recorded $\mathrm{Lp}$ (a) concentration and subsequent major vascular morbidity and/or cause-specific mortality published between 1970 and 2009 were analyzed [20]. Thirty-six eligible prospective studies were identified, including a total of 126,634 individuals who had no known prior history of CHD or stroke at the baseline examination. During a median of 9.8 (IQR 3.5-21.3) years comprising 1.3 million person years at risk, the association of $\mathrm{Lp}(\mathrm{a})$ with fatal vascular and non-vascular outcomes was analyzed. Hazard ratios and odds ratios were described as risk ratios (RRs) using Cox proportional hazard regression models. RRs and 95\% CI per 3.5-fold higher usual Lp(a) levels adjusted for cardiovascular risk factors were 1.15 (CI 1.08, 1.22) for coronary death, 1.20 (CI 1.10, 1.30) for fatal MI, and 1.10 (CI 1.06, 1.14) for all vascular deaths. No risk association was found with non-vascular death.

The Brisighella Heart Study (BHS) is a prospective, population-based longitudinal epidemiological investigation, since 1972 involving 2939 randomly selected subjects aged 14 to 84 years, resident in the northern Italian rural town of Brisighella, and free from ASCVD at enrollment [21]. For cardiovascular-risk groups a receiver operat- ing characteristic curve (ROC curve) was used to assess whether the $\mathrm{Lp}$ (a) concentration was an independent longterm prognostic factor for cardiovascular mortality based on a follow-up of 25 years. The $\mathrm{Lp}$ (a) concentration appeared with limited strength as a predictive test of longterm mortality in subjects with a high cardiovascular risk profile (AUC $=0.63,95 \%$ CI [0.50-0.76], $p=0.049$ ) [21]. The matchable Kuopio Ischemic Heart Disease study followed a cohort of 1881 Finnish men aged 42-61 years also over a median period of 24.7 years [22]. Cumulative hazard curves demonstrated a greater risk of sudden cardiac death, which is a particular aspect of cardiovascular mortality in the top quartile of $\mathrm{Lp}(\mathrm{a})$ levels compared to those in the bottom quartile ( $p=0.032$ for log-rank test).

Mortality was examined in white individuals of Danish descent from the Copenhagen City Heart Study (CCHS) and the Copenhagen General Population Study (CGPS) [23]. The CCHS was initiated in 1976-1978, the CGPS was initiated in 2003-2014. High levels of Lp(a) through corresponding low LPA-Kringle IV-type 2 number of repeats were associated with high risk of both cardiovascular and total mortality, but not non-cardiovascular mortality. Compared with individuals with $\mathrm{Lp}(\mathrm{a})$ levels $<10 \mathrm{mg} / \mathrm{dl}$ (1st-50th percentiles), the multivariable adjusted hazard ratio of cardiovascular mortality was 1.50 (95\% CI 1.28-1.76) for individuals with $\mathrm{Lp}$ (a) levels $>93 \mathrm{mg} / \mathrm{dl}$ (96th-100th percentiles), 1.32 (1.12-1.56) for $69-93 \mathrm{mg} / \mathrm{dl}$ (91st-95th percentiles), 1.02 (0.89-1.16) for $43-68 \mathrm{mg} / \mathrm{dl}$ (81st-90th percentiles), and 0.97 (0.89-1.07) for individuals with $\mathrm{Lp}$ (a) levels of $10-42 \mathrm{mg} / \mathrm{dl}$ (51st-80th percentiles). The corresponding hazard ratios for all-cause mortality were 1.20 (1.10-1.30), 1.07 (0.98-1.17), 1.01 (0.94-1.08), and 0.97 (0.92-1.01), respectively.

In two investigations $\mathrm{Lp}$ (a) concentrations did not impact mortality overall, but an interaction between increasing $\mathrm{Lp}$ (a) concentrations and diabetes mellitus (Dm) was observed [24, 25]. Results were not yet influenced by the putative effect of sodium-glucose co-transporter 2 inhibition on cardiovascular mortality in high-risk patients with Dm. In 700 patients with peripheral artery disease presence of Dm, in particular if insulin-dependent and Lp(a) values in the highest tertile $(>36 \mathrm{mg} / \mathrm{dl})$ were associated with an increased risk for death [24]. This association remained consistent adjusting for other cardiovascular risk factors and use of lipid-lowering medication, revealing an approximately 3-fold increased adjusted risk in patients with insulin-dependent type II Dm compared to non-diabetics. Cox proportional hazards models revealed adjusted hazard ratios of $1.33(95 \% \mathrm{CI} 0.88-1.97, p=0.11)$ and 1.62 (95\% CI 1.10-2.45, $p=0.019)$ for patients with non-insulindependent and insulin-dependent type II Dm, respectively. This significant interaction remained essentially identical for total as well as cardiovascular mortality. As part of the 
Biomarkers for Cardiovascular Risk Assessment in Europe (BiomarCaRE) project data of 56,804 participants without a prior history of major ASCVD from 7 prospective population-based cohorts across Europe failed to show a significant association between $\mathrm{Lp}(\mathrm{a})$ levels and total mortality [25]. However, in the predefined subgroup of individuals with $\mathrm{Dm}$, the Lp(a)-associated HR for total mortality was 1.15 compared to individuals without $\mathrm{Dm}$ [25].

Between 1997 and 2000, 3313 German patients were enrolled after coronary angiography, including $78 \%$ with established CHD, in the Ludwigshafen Risk and Cardiovascular Health (LURIC) study [26]. Associations of tertiles of $\mathrm{Lp}$ (a) concentration and two LPA single-nucleotide polymorphisms ([SNPs] rs10455872 and rs3798220) with all-cause mortality and cardiovascular mortality were tested by Cox regression analysis. Increased severity of coronary heart disease was associated with $\operatorname{Lp}(\mathrm{a})$ concentrations in the highest tertile (adjusted hazard radio [HR] 1.44, 95\% CI 1.14-1.83) and the presence of either LPA SNP (1.88, 95\% CI 1.40-2.53). At the level of aggregated results five independent studies well matched for inclusion criteria and available cardiovascular endpoints (Table 1) were compared [26]. No associations were found for $\operatorname{Lp}(a)$ with all-cause mortality or cardiovascular mortality in LURIC or in the validation studies [26].

The above summarized studies encompass a wide range of cohort features, sizes, and statistical analyses potentially including confounders of trial-related differences. There are a number of potential general confounders (e.g. restrictive inclusion/exclusion criteria of RCTs, lack of statistical power, index event bias, differences in clinical management between primary and secondary prevention), as well as $L p$ (a) related confounders (e.g. use of log-transformed $\mathrm{Lp}$ (a) concentrations, changes in $\mathrm{Lp}(\mathrm{a})$ concentrations due to acute events, $\mathrm{Lp}(\mathrm{a})$ assay standardization, size isoformdependent bias in Lp(a) measurement, effect of sample handling and storage on $\mathrm{Lp}(\mathrm{a})$ ) [27]. None of these investigations or studies included cohorts of patients who had been selected to assess Lp(a) effects on ASCVD. The use of aggregated data sets rather than individual participant data from each study might create uncontrollable bias potentially masking $\mathrm{Lp}(\mathrm{a})$ associated effects in general or in particular subgroups. Given the highly positively skewed distribution of $L p(a)$ concentrations, many studies may be underpowered to detect an association between the upper range of $\mathrm{Lp}$ (a) concentrations and ASCVD events. If the goal of a study is to identify situations where $\mathrm{Lp}$ (a) lowering might confer a clinical benefit, it is reasonable to enroll a cohort with higher median $\mathrm{Lp}$ (a) levels than the general population. The German reimbursement guideline for the indication of LA in patients with Lp(a)-HLP has proved to be of value to identify patients at highest risk using the composite of a $\mathrm{Lp}(\mathrm{a})$ threshold $>60 \mathrm{mg} / \mathrm{dl}(120 \mathrm{nmol} / \mathrm{l})$ and clinical ASCVD progression despite effective LDL-C lowering therapy [11].

\section{Conclusion}

By the majority of existing investigations an association of $\mathrm{Lp}$ (a) concentration on total or cardiovascular mortality was demonstrated. However, inconsistency in the findings between studies exists without a trend for any study feature to explain this. Genetic homogeneity of the population, longterm follow-up, and clinically guided selection of patients might be important to further clarify the impact of $L p(a)$ concentration on progression of ASCVD, and finally total or cardiovascular mortality. LDL and Lp(a) particles exhibit a mutual effect modification on related ASCVD risk. Therefore, LDL-C levels and concomitant LDL-C lowering treatment must be considered. Prospective evaluation is needed to document that specific $\mathrm{Lp}$ (a)-lowering additional to targeted LDL-C lowering will in fact reduce cardiovascular or total mortality.

\section{Compliance with ethical guidelines}

Conflict of interest R. Klingel, A. Heibges and C. Fassbender are affiliates of the Apheresis Research Institute, which received financial support for clinical research activities by grants from Asahi Kasei Medical, Japan and Diamed, Germany.

Ethical standards This article does not contain any studies with human participants or animals performed by any of the authors.

Open Access This article is distributed under the terms of the Creative Commons Attribution 4.0 International License (http:// creativecommons.org/licenses/by/4.0/), which permits unrestricted use, distribution, and reproduction in any medium, provided you give appropriate credit to the original author(s) and the source, provide a link to the Creative Commons license, and indicate if changes were made.

\section{References}

1. Kostner G, Avogaro P, Cazzolato G et al (1981) Lipoprotein Lp(a) and the risk of myocardial infarction. Atherosclerosis 38:51-61

2. Nordestgaard BG, Langsted A (2016) Lipoprotein(a) as a cause of cardiovascular disease: insights from epidemiological, genetics, and biology. J Lipid Res 57:1953-1975

3. Kamstrup PR, Benn M, Tybjaerg-Hansen A, Nordestgaard BG (2008) Extreme lipoprotein(a) levels and risk of myocardial infarction in the general population. The Copenhagen City Heart Study. Circulation 117:176-184

4. Kronenberg F, Utermann G (2013) Lipoprotein(a)—resurrected by genetics. J Intern Med 273:6-30

5. Tsimikas S, Fazio S, Ferdinand KC et al (2018) NHLBI working group recommendations to reduce lipoprotein(a)-mediated risk of cardiovascular disease and aortic stenosis. J Am Coll Cardiol 71:177-192

6. Verbeek R, Hoogeveen RM, Langsted A et al (2018) Cardiovascular disease risk associated with elevated lipoprotein(a) attenuates at low 
low-density lipoprotein cholesterol levels in a primary prevention setting. Eur Heart J 39:2589-2596

7. Nordestgaard BG, Chapman MJ, Ray K et al (2010) Lipoprotein(a) as a cardiovascular risk factor: current status. Eur Heart J 31:2844-2853

8. Chen HY, Dufresne L, Burr H et al (2018) Association of LPA variants with aortic stenosis. A large scale study using diagnostic and procedural codes from electronic health records. JAMA Cardiol 3:18-23

9. Desmarais RL, Sarembock IJ, Ayers CR et al (1995) Elevated serum Lipoprotein(a) is a risk factor for clinical recurrence after coronary balloon angioplasty. Circulation 91:1403-1409

10. Zairis MN, Ambrose JA, Manousakis SJ et al (2002) The impact of plasma levels of C-reactive protein, Lipoprotein (a) and homocysteine on the long-term prognosis after successful coronary stenting. J Am Coll Cardiol 40:1375-1382

11. Roeseler E, Julius U, Heigl F et al, Pro(a)LiFe-study group (2016) Lipoprotein apheresis for lipoprotein(a)-associated cardiovascular disease: prospective 5 years of follow-up and apolipoprotein(a) characterization. Arterioscler Thromb Vasc Biol 36:2019-2027

12. Navar AM, Peterson ED (2018) Challenges in interpreting the lipidlowering trials biology vs ecology. JAMA 319:1549-1551

13. Cannon CP, Blazing MA, Giugliano RP et al (2015) Ezetimibe added to statin therapy after acute coronary syndromes. N Engl J Med 372:2387-2397

14. Navarese EP, Robinson JG, Kowalewski M et al (2018) Association between baseline LDL-C level and total and cardiovascular mortality after LDL-C lowering. A systematic review and meta-analysis. JAMA 319:1566-1579

15. Sabatine MS, Giugliano RP, Keech AC et al (2017) Evolocumab and clinical outcomes in patients with cardiovascular disease. N Engl J Med 376:1713-1722

16. Willeit P, Ridker PM, Nestel PJ et al (2018) Baseline and onstatin treatment lipoprotein(a) levels for prediction of cardiovascular events: individual patient-data meta-analysis of statin outcome trials. Lancet. https://doi.org/10.1016/s0140-6736(18)31652-0
17. Perrot N, Verbeek R, Sandhu M et al (2017) Ideal cardiovascular health influences cardiovascular disease risk associated with high lipoprotein(a) levels and genotype: the EPIC-Norfolk prospective population study. Atherosclerosis 256:47-52

18. Berg K, Dahlen G, Christophersen B et al (1997) Lp(a) lipoprotein level predicts survival and major coronary events in the Scandinavian Simvastatin Survival Study. Clin Genet 52:254-261

19. Ariyo AA, Thach C, Tracy R for the Cardiovascular Health Study Investigators (2003) Lp(a) lipoprotein, vascular disease, and mortality in the elderly. N Engl J Med 349:2108-2015

20. Emerging Risk Factors Collaboration, Erqou S et al (2009) Lipoprotein(a) concentration and the risk of coronary heart disease, stroke, and nonvascular mortality. JAMA 302:412-423

21. Fogacci F, Cicero AFG, D'Addato S et al (2017) Serum lipoprotein(a) level as long-term predictor of cardiovascular mortality in a large sample of subjects in primary cardiovascular prevention: data from the Brisighella Heart Study. Eur J Int Med 37:49-55

22. Kunutsor SK, Khan H, Nyyssönen K et al (2016) Lipoprotein(a) and risk of sudden cardiac death in middle-aged Finnish men: a new prospective cohort study. Int J Cardiol 220:718-725

23. Langsted A, Kamstrup PR, Nordestgaard BG (2019) High lipoprotein(a) and high risk of mortality. Eur Heart J. https://doi.org/10. 1093/eurheartj/ehy902

24. Maca T, Mlekusch W, Doweik L et al (2007) Influence and interaction of diabetes and lipoprotein (a) serum levels on mortality of patients with peripheral artery disease. Eur J Clin Invest 37:180-186

25. Waldeyer C, Makarova N, Zeller T et al (2017) Lipoprotein(a) and the risk of cardiovascular disease in the European population: results from the BiomarCaRE consortium. Eur Heart J 38:2490-2498

26. Zewinger S, Kleber ME, Tragante V et al (2017) Relations between lipoprotein(a) concentrations, LPA genetic variants, and the risk of mortality in patients with established coronary heart disease: a molecular and genetic association study. Lancet Diabetes Endocrinol 5:534-543

27. Boffa MB, Stranges S, Klar N et al (2018) Lipoprotein(a) and secondary prevention of atherothrombotic events: a critical appraisal. J Clin Lipidol 12:1358-1366 